\title{
Evaluation of Different Media for the Production of Cephalosporins by Streptomyces clavuligerus ATCC 27064
}

\author{
Tatiana Antonio ${ }^{1}$, Carolina Bellão ${ }^{2}$, Tatiana Corrêa ${ }^{1}$, André Pastrelo Cavallieri ${ }^{1}$, Alberto \\ Colli Badino ${ }^{2}$ and Maria Lucia Gonsales da Costa Araujo ${ }^{1 *}$ \\ ${ }^{I}$ Departamento de Bioquímica e Química Tecnológica; Instituto de Química; Universidade Estadual Paulista; \\ 14800-900; Araraquara - SP - Brasil. ${ }^{2}$ Departamento de Engenharia Química, Universidade Federal de São \\ Carlos; 13565-905; São Carlos - SP - Brasil
}

\begin{abstract}
The aim of this work was to compare the composition of a complex and soluble culture medium to eight other media described in the literature through the batch cultivation in a conventional bench-scale bioreactor for the production of cephamycin $C$ by a wild strain of Streptomyces clavuligerus. The proposed medium resulted in an antibiotic production 1.5 to 7.5 times higher than the other culture media.
\end{abstract}

Key words: $\beta$-lactam antibiotic, Streptomyces clavuligerus, lysine metabolism, complex medium

\section{INTRODUCTION}

Various $\beta$-lactam compounds are produced by Streptomyces clavuligerus in submerged culture, which include the antibiotics of the cephalosporin $\mathrm{C}$ biosynthetic route, such as cephamycin $\mathrm{C}$ (CephC), and clavulanic acid (CA), a powerful $\beta$ lactamases inhibitor (Liras and Martín 2005). The cephalosporins and CA biosynthetic pathways are completely different from each other, and like most bioactive compounds, they are rigorously controlled by the intra-and extra-cellular factors (Santamarta et al. 2005). The presence of certain nutrients in the culture medium, such as the sources of C, N, P, or sulfur S, and salts, as well as the substances of the biosynthetic route can promote the production of CA or CephC (Bussari et al. 2008). Glycerol is preferably used as $C$ source for the production of CA, whereas starch is more suitable for the production of CephC (Zhu et al. 2007; Sánchez et al. 2010).
Organic sources of $\mathrm{N}$, such as amino acids and protein extracts, are more appropriate for the growth of $S$. clavuligerus than the inorganic ones and they should be carefully selected since they exert a strong influence on the secondary metabolism (Masurekar 2008). The positive effects of adding ornithine or high concentrations of lysine in synthetic culture medium of $S$. clavuligerus for the synthesis of CA or CephC, respectively, have been emphasized (Chen et al. 2003; Domingues et al. 2009). Several studies have shown that sulfur atom from the inorganic sources is more efficiently incorporated into the CephC molecule than sulfur from methionine or cysteine (Bussari et al. 2008). Nevertheless, the potential benefit of adding such compounds in the complex media with the aim of improving the antibiotic production has been suggested only in the technical publications, such as patents (EgelMitani et al. 1999). In this work, a complex and soluble culture medium was evaluated for the

*Author for correspondence: mlaraujo@iq.unesp.br 
production of cephalosporins by the wild strain of S. clavuligerus, and its performance was compared to eight other culture media described in the literature in batch cultivations in a conventional bench scale bioreactor.

\section{MATERIALS AND METHODS}

Vegetative cells $(8.0 \mathrm{~g} /$ /iter $)$ of $S$. clavuligerus ATCC 27064 were stored in cryotubes at $-70^{\circ} \mathrm{C}$ using glycerol $(20 \% \mathrm{v} / \mathrm{v})$. The reactivation medium contained $(\mathrm{g} / \mathrm{l})$ : tryptone 5 , yeast extract 3 , and malt extract 10 . The basal inoculum medium was composed of (in $\mathrm{g} / \mathrm{l}$ ): yeast extract 1 , malt extract $10, \mathrm{~K}_{2} \mathrm{HPO}_{4} 0.8, \mathrm{MgSO}_{4} \cdot 7 \mathrm{H}_{2} \mathrm{O} 0.75$, and salt solution $10 \mathrm{ml} / \mathrm{l}$ containing (in $\mathrm{g} / \mathrm{l}$ ): $\mathrm{MnCl}_{2} \cdot 4 \mathrm{H}_{2} \mathrm{O} 1, \mathrm{FeSO}_{4} \cdot 7 \mathrm{H}_{2} \mathrm{O} 1$, and $\mathrm{ZnSO}_{4} \cdot 7 \mathrm{H}_{2} \mathrm{O}$ 1. The basal production medium contained (in $\mathrm{g} / \mathrm{l}$ ): yeast extract $0.5, \mathrm{~K}_{2} \mathrm{HPO}_{4} 1.75, \mathrm{MgSO}_{4} \cdot 7 \mathrm{H}_{2} \mathrm{O}$ $0.75, \mathrm{CaCl}_{2} .2 \mathrm{H}_{2} \mathrm{O} 0.2, \mathrm{NaCl} 2$, sodium thiosulfate 1 , added at $30 \mathrm{~h}$ post-inoculation according to Inamine and Birnbaum (1976), and salt solution described above $(5 \mathrm{ml} / \mathrm{l})$. The media were supplied with $21 \mathrm{~g} / \mathrm{l}$ of the buffering agent 3-(Nmorpholino) propanesulfonic acid (MOPS), and the initial $\mathrm{pH}$ was adjusted to 6.8 .

In order to avoid the excessive increase in viscosity of the broth and the problems related to oxygen limitation during the shake-flask process, the broth volume was $10 \%(\mathrm{v} / \mathrm{v})$ in relation to the nominal volume of the flask, and the concentration of the $\mathrm{C}$ sources (glycerol and/or starch) was established to reach a total of $10 \mathrm{~g} / \mathrm{l}$, considering a mean cell yield coefficient based on substrate consumption of $\mathrm{Y}_{\mathrm{X} / \mathrm{S}}=0.6 \pm 0.1 \mathrm{~g} / \mathrm{g}$ (Gouveia et al. 2001). The main $\mathrm{N}$ sources were composed of cottonseed extract (Proflo, Traders Protein), casein hydrolysate enzymatic, casein hydrolysate acid, or soybean protein hydrolysate (Soytone Bacto ${ }^{\mathrm{TM}}$, BD) - at a mass ratio of $\mathrm{C}: \mathrm{N}=5: 1$, and $100 \mathrm{mM}$ of L-lysine. The final concentration of the $\mathrm{N}$ sources resulted in a mass proportion of $\mathrm{C}: \mathrm{N}=1.4: 1$ in the production medium.

Fermentation runs were carried out in shake flasks $\left(500 \mathrm{ml}\right.$ nominal volume, $260 \mathrm{rpm}, 28^{\circ} \mathrm{C}$ ) at $10 \%$ $(\mathrm{v} / \mathrm{v}$, total volume per nominal volume), in triplicate. In bench scale bioreactors (New Brunswick Bioflo 2000, 51 working volume), the runs were performed at $\mathrm{pH} 6.8$ and $28^{\circ} \mathrm{C}$ and dissolved oxygen (DO) level was controlled at
$40 \%$ of air saturation by varying the stirrer speed at specific air flow rate of $1 \mathrm{vvm}$. The main fermentation inoculum was obtained from two subsequent cultures (ca. $24 \mathrm{~h}$ each) in the reactivation and inoculum media, respectively, maintaining a $10 \%(\mathrm{v} / \mathrm{v})$ inoculum ratio. The concentration of CephC was expressed in terms of the total cephalosporins' concentration, which was determined by agar diffusion bioassay using Escherichia coli ESS 2235 and cephalosporin C as standard (Liras and Martín 2005). Penicillin N was removed by adding $0.25 \mu \mathrm{l} / \mathrm{ml}$ of sample of $\mathrm{BD}$ Difco $^{\mathrm{TM}}$ Penase (power $1977 \mathrm{UI} / \mathrm{min} / \mathrm{ml}, \mathrm{pH} 7$, $25^{\circ} \mathrm{C} ; 1 \mathrm{UI} \equiv 6.27 \times 10^{-4} \mathrm{mg}$ of PenG), followed by reaction at $25^{\circ} \mathrm{C}$ for $20 \mathrm{~min}$.

Biomass was determined as DCW/1 $\left(105^{\circ} \mathrm{C}\right.$, overnight). Glycerol was determined as formaldehyde after reaction of the samples with periodic acid. Starch was quantified as total reducing sugars by using the DNS method, after hydrolysis (HCL $10 \mathrm{M}$ ). Lysine was determined by the post column orthophthaldialdehyde (OPA) derivatization method. Enzymatic activity of lysine $\varepsilon$-aminotransferase (LAT) and cadaverine aminotransferase (CAT) was determined according to Khethan et al. (1999).

\section{RESULTS}

Figures $1 \mathrm{~A}$ and $1 \mathrm{~B}$ show the consumption of glycerol and starch in culture media containing different proportions of these $\mathrm{C}$ sources, and cottonseed extract (Proflo) as complex N source. The initial content of phosphate $(10 \mathrm{mM})$ was low enough to avoid the repression and/or inhibition of the synthesis of antibiotics (Martín 2004). The cell concentration was similar in all the media, and the highest production of cephalosporins was obtained in the medium containing only starch (Figs. 2A and $2 \mathrm{~B}$ ).

Therefore, four media containing different complex and soluble $\mathrm{N}$ sources were tested using starch as the main source of $\mathrm{C}$ and energy. Figures $3 \mathrm{~A}$ and $3 \mathrm{~B}$ show the time profiles of the biomass and production of total cephalosporins obtained in these cultures. The cottonseed extract (Proflo) led to a higher production of the antibiotic, and thus it was used as the complex $\mathrm{N}$ source in the media in the subsequent experiments. 

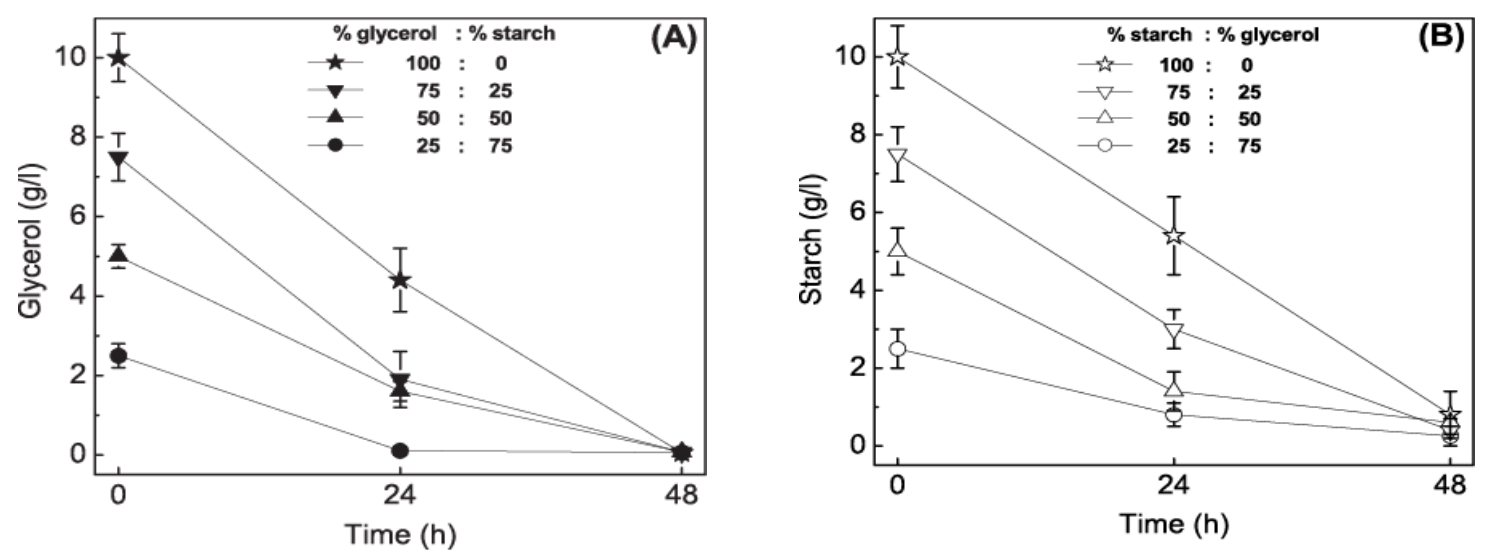

Figure 1 - Profile of consumption of glycerol (A) and starch (B) in shake flasks experiments using cottonseed extract as $\mathrm{N}$ source.
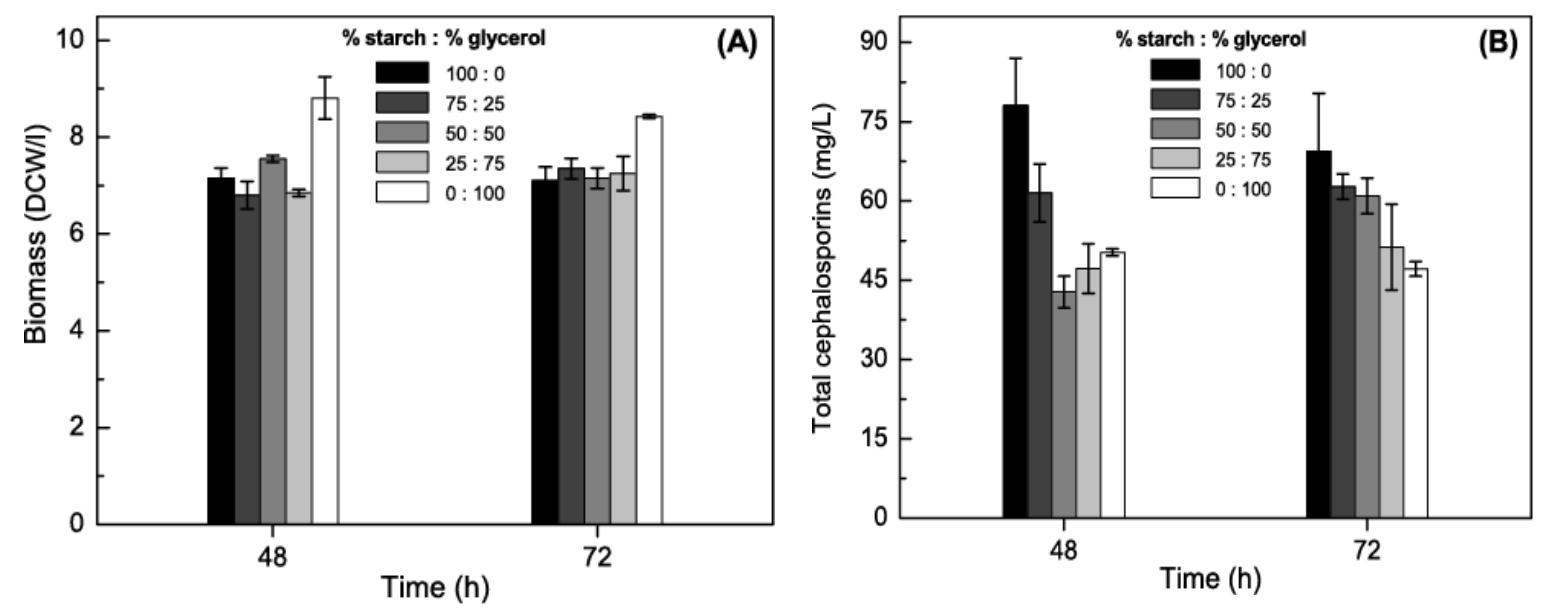

Figure 2 - Biomass (A) and total cephalosporins (B) in shake flasks experiments.
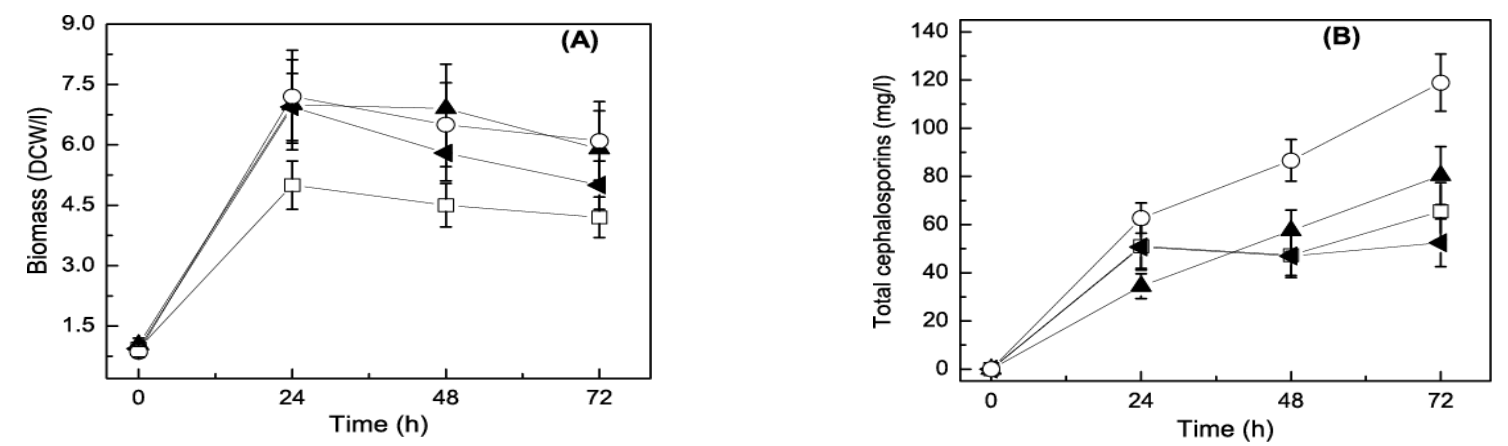

Figure 3 - Biomass (A) and total cephalosporins (B) in shake flasks experiments using starch, Llysine, and cottonseed extract $(\bigcirc)$, soybean protein hydrolysate $(\boldsymbol{\Delta})$, casein hydrolysate acid ( $\square)$, and triptone $(\varangle)$.

The concentration of $100 \mathrm{mM}$ lysine initially used in the production medium was based on the best results obtained by Fang et al. (1996) in synthetic medium cultures. Increases in the production of cephalosporins by $S$. clavuligerus in the presence of high concentrations of exogenous lysine were first reported by these authors. In order to establish an appropriate initial concentration of lysine in the proposed medium, the cultures were carried out varying the concentration from 10 to $150 \mathrm{mM}$ 
amino acid. Figures 4A and 4B show the concentration-time profiles of cell and antibiotics, respectively. The activities of CAT and LAT measured in samples withdrawn at $24 \mathrm{~h}$ of fermentation are shown in Figure 4C, and Figure 4D shows the consumption of lysine.

Figure 5 shows the activity of LAT and CAT
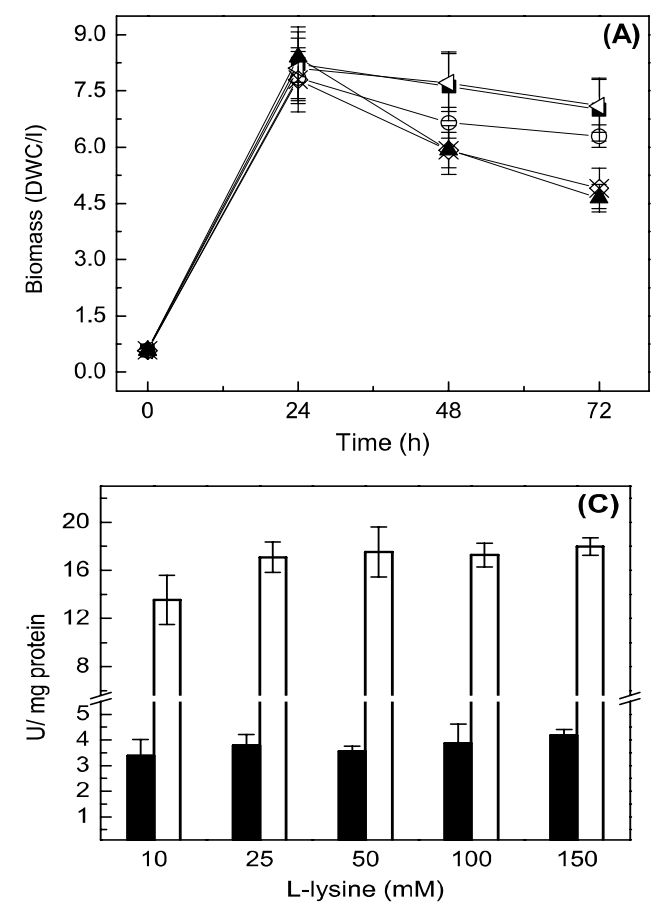

measured in the samples withdrawn at different time intervals during the fermentations carried out with culture media initially containing 10 and 100 $\mathrm{mM}$ lysine. Figures $6 \mathrm{~A}$ and $6 \mathrm{~B}$ show the concentration- time profiles of $\mathrm{C}$ sources and total cephalosporins, respectively, in all the tested media.
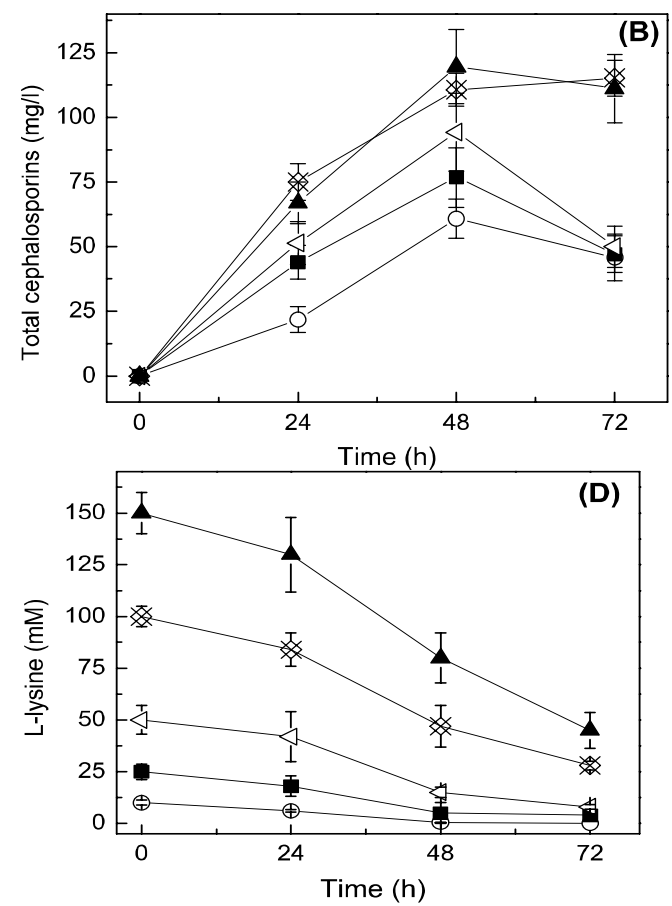

Figure 4 - Biomass (A), total cephalosporins (B), enzymatic activity - LAT (solid columns) and CAT (open columns) (C), and profile of consumption of L-lysine (initial concentration, in mM): $10(\mathrm{O}), 25(\mathbf{\square}), 50(\varangle), 100(\Varangle), 150(\mathbf{\Lambda})(\mathrm{D})$, in shake flasks experiments.

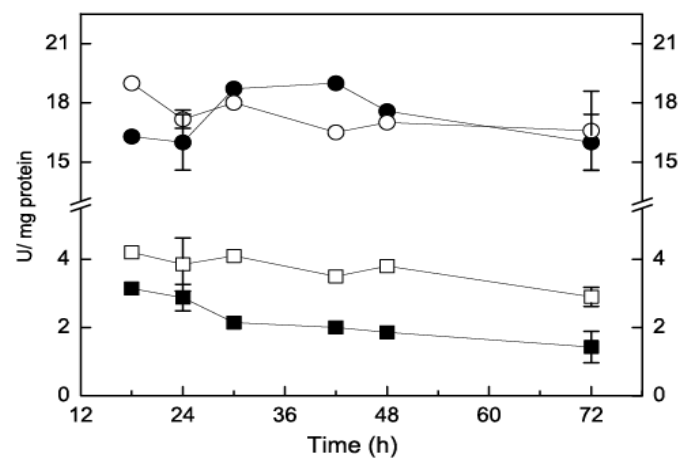

Figure 5 - Enzymatic activity of - LAT (square) and CAT (circle) -in shake flasks experiments with initial concentrations of $10 \mathrm{mM}$ (solid symbols) and $100 \mathrm{mM}$ (open symbols) Llysine. 

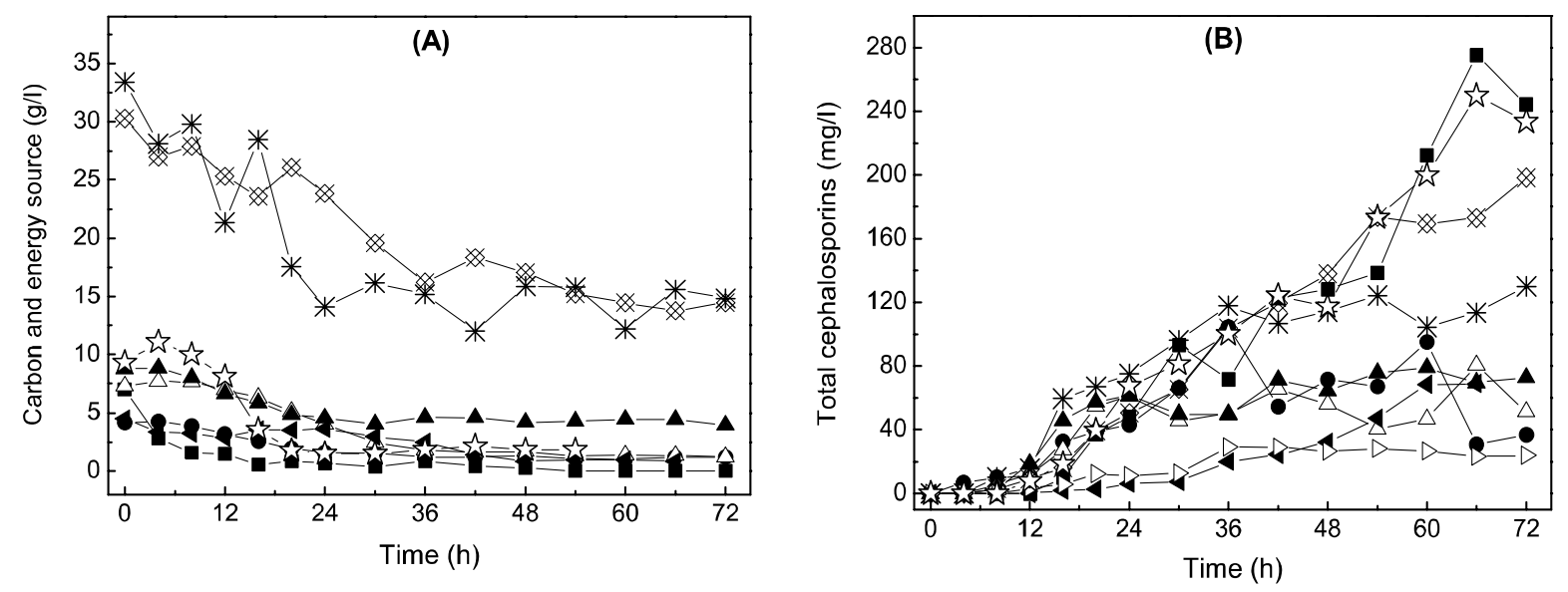

Figure 6 - Main C source consumption (A) and total cephalosporins (B) in batch cultivation in agitated and aerated bench-bioreactor in the cephamycin $\mathrm{C}$ production media (initial concentration of sources of C and energy, and N, in g/l): Park et al. (1994) (soybean oil, 7; soybean flour, 20; casamino acid, 10; lysine, 1) (घ); Dévi and Sridhar (1999) (soluble starch, 5; corn steep liquor, 5; sunflower oil cake, 2.5) (•); Sarada and Sridhar (1998) (cassava flour, 10; corn steep liquor, 10; sunflower oil cake, 5) ( $\triangle$ ); Rollins et al. (1988a) (soluble starch, 10; tryptone, 17; peptone, 3) (A); Kamogashira et al. (1982) (soluble starch, 30, and sucrose, 10; cottonseed extract, 20; dry yeast, 10 ) (*); Aharonowitz et al. (1978) (soluble starch, 5; asparagine, 2) (4); Park et al. (1994) (soybean oil, 30; peptone, 5; $\left.\left(\mathrm{NH}_{4}\right)_{2} \mathrm{SO}_{4}, 1\right)(\triangleright)$; Kamogashira et al. (1982), modified by the addition of $5 \mathrm{~g} / \mathrm{l}$ of sodium thiosulphate (soluble starch, 30; sucrose, 10; cottonseed extract, 20; dry yeast, 10; lysine, 1) ( this study (soluble starch, 10.0; cottonseed extract, 8.5; lysine ,18.3) (绽).

\section{DISCUSSION}

In this work, a complex soluble production medium was chosen, which presented advantages in relation to culture media with insoluble components, mainly the easier control of operating variables and downstream processing. The best results in terms of cephalosporin production were obtained using starch as the main source of $\mathrm{C}$ and energy. It is consistent with various studies which have suggested that the continuous and gradual hydrolysis of starch can avoid mechanisms of repression and /or inhibition in the production of $\beta$-lactam antibiotics that are normally triggered by $\mathrm{C}$ sources that are more easily metabolized by the microorganism, such as glucose or glycerol (Alexander et al. 2007; Sánchez et al. 2010). It has been observed that these easily assimilated sources can lead to an accumulation of intracellular phosphorylated compounds that drastically inhibit the expandase activity, which is the enzyme responsible for the expansion of the penicillin $\mathrm{N}$ ring of the $\beta$-lactam antibiotics biosynthetic pathway (Martín 2004; Zhu et al. 2007).
Moreover, the negative effect of the excess of glycerol on antibiotic production is more evident when this substrate is added at the beginning of the fermentation, both alone or with starch.

At first glance, the results of lysine consumption obtained in the present work were inconsistent with the need to add high concentrations of that amino acid to provide significant increases in the production of the antibiotic, as suggested in the literature. This was justified based on the higher demand of lysine in the primary metabolism (via CAT), when compared to the demand required for the antibiotic biosynthetic route (via LAT), in which the amino acid flow was much less favorable (Leitão et al. 2001). Earlier studies have demonstrated significant increases in the production (200 to $500 \%$ in relation to medium without lysine) and significant effects on the cell growth in synthetic media containing 50 to 150 $\mathrm{mM}$ of lysine. Fang et al.(1996) evaluated the production of cephalosporins in synthetic medium containing from 50 to $150 \mathrm{mM}$ of lysine and obtained the highest production in the medium with $100 \mathrm{mM}$ amino acid, around $125 \mathrm{mg}$ 
cephalosporins $/$, which is approximately $500 \%$ higher than that obtained without lysine.

The LAT activity seemed to be independent of the initial lysine concentration in the culture media, presenting the same level of expression in $24 \mathrm{~h}$ of fermentation. These results were consistent with the previous data from the literature which demonstrated that LAT was preferably synthesized in the exponential growth phase and presented higher activity at this stage of the process (Khethan et al. 1999). However, in addition to playing the role of positive regulator of the LAT expression, it was observed that the excess of lysine in the medium, initially containing $100 \mathrm{mM}$ of amino acid, supported the maintenance of the LAT activity at slightly higher values along the fermentation, when compared with those observed in $10 \mathrm{mM}$ supplemented medium. This suggested that the excess of lysine provided an increase in the enzyme loading of the cell, thus leading to more intracellular concentration of the enzymesubstrate complex, when compared to that observed in the low amino acid concentration media. It could be worth mentioning that the conditions of the analytical method used were established to provide the maximum activity (in vitro) of the sample enzymes. Hence, the substrate excess, the $\mathrm{pH}$ values, and suitable temperatures, as well as a period of time during which the maximum reaction rate was kept, were guaranteed. These conditions, besides the fact that the cell concentration of the suspension from which the protein material was extracted, was the same in all the samples, enabled concluding that the difference in the results of the samples were due to the difference in the enzyme loading of the cell. On the other hand, CAT activity was quite stable along the fermentations using both 10 and 100 $\mathrm{mM}$ of lysine.

With relation to the comparison between the proposed medium and other culture media described in the literature, the maximum value of antibiotic yield based on substrate consumption $\left(\mathrm{Y}_{\mathrm{P} / \mathrm{S}}\right)$ was obtained by Park et al. (1994). However, in this medium, the soy oil should not be considered as the only source of $\mathrm{C}$ and energy in this culture medium, since it also contained $20 \mathrm{~g} / \mathrm{l}$ of soy flour, which presented ca. $38 \%(\mathrm{w} / \mathrm{w})$ carbohydrate in its composition. Therefore, the medium used by Park et al. (1994) and proposed medium were the most suitable for cephalosporins production among the tested media.
The results showed that although limited by the productive capacity of a wild-strain microorganism, the manipulation of the medium formulation in order to increase the production of a specific metabolite could be still an efficient method to intervene in the strain performance.

\section{ACKNOWLEDGMENTS}

The authors are grateful for the financial support provided by the foundations FAPESP (Fundação de Apoio a Pesquisa do Estado de São Paulo (Processes 05/55079-4, 08/03579-1, 08/52819-5), and CAPES (Coordenação de Aperfeiçoamento de Pessoal de Nivel Superior).

\section{REFERENCES}

Aharonowitz Y, Demain AL. Carbon catabolite regulation of cephalosporin production in Streptomyces clavuligerus. Antimicrob Agents Chemother. 1978; 14:159-164.

Alexander DC, Anders CL, Lee L, Jensen SE. pcd mutants of Streptomyces clavuligerus still produces cephamycin C. J Bacteriol. 2007; 189:5867-5874.

Bussari B, Saudagar PS, Shaligram NS, Survase SA, Singhal RS. Production of cephamycin C by Streptomyces clavuligerus NT4 using solid-state fermentation. J Ind Microbiol Biotechnol. 2008; 35: 49-58.

Chen KC, Lin YH, Wu JY, Hwang SCJ. Enhancement of clavulanic acid production in $\mathrm{S}$. clavuligerus with ornithine feeding. Enzyme Microbial Technol. 2003; 32:152-156.

Dévi S, Sridhar P. Optimization of critical parameters in immobilization of Streptomyces clavuligerus on alginate gel matrix for cephamycin $\mathrm{C}$ production. World J Microbiol Biotechnol. 1999; 15:185-192

Domingues LCG, Teodoro JC, Hokka CO, Badino AC, Araujo MLGC. Optimisation of the glycerol-toornithine molar ratio in the feed medium for the continuous production of clavulanic acid by Streptomyces clavuligerus. Biochem Eng. J. doi:10.1016/j.bej.2009.05.006

Egel-Mitani M, Kaasgaard S, Kristiansen KN. Process for the production of secondary metabolites. U.S. Pat. 1999 March (5), 882-883.

Fang A, Keables, P, Demain AL. Unexpected enhancement of $\beta$-lactam antibiotic formation in Streptomyces clavuligerus by very high concentrations of exogenous lysine. Appl Microbiol Biotechnol. 1996; 44:705-709. 
Gouveia ER, Baptista Neto A, Badino AC, Hokka CO. Optimization of medium composition for clavulanic acid production by Streptomyces clavuligerus. Biotechnol Lett. 2001; 23:157 -161.

Inamine $\mathrm{E}$, Birnbaum J. Cephamycin $\mathrm{C}$ by fermentation. U.S. Pat. 1976 August (3), 977-942.

Kamogashira T, Nishida T, Sugawara M, Nihno T, Takegata S. Process for the production of antibiotic cephamycin C. U.S. Pat.1982 June (4), 332-891.

Khethan A, Malberg LH, Kyung YS, Sherman DH, Hu WS. Precursor and cofactor as a check valve for cephamycin biosynthesis in Streptomyces clavuligerus. Biotechnol Prog. 1999; 151:020 -1027.

Leitão AL, Enguita FJ, Martín JF, Oliveira JFS. Effect of exogenous lysine on the expression of early cephamycin $\mathrm{C}$ biosynthetic genes and antibiotic production in Nocardia lactamdurans MA4213. Appl Microbiol Biotechnol. 2001; 56: 670-675.

Liras P, Martín JF. Microbial Processes and Products. Humana Press, New Jersey: Barredo JL. (ed); 2005.

Martín JF. Phosphate control of the biosynthesis of antibiotics and other secondary metabolites is mediated by the PhoR-PhoP system: an unfinished story. J Bacteriol. 2004; 186: 5197 -5201.

Masurekar PS. Nutritional and engineering aspects of microbial process development. Prog Drug Res. 2008; 65:291-328.

Park YS, Momose I, Tsunoda K, Okabe M. Enhancement of cephamycin $\mathrm{C}$ production using soybean oil as the sole carbon source. Appl Microbiol Biotechnol. 1994; 40: 773-779.
Rollins MJ, Jensen SE, Westlake DWS. Effect of aeration on antibiotic production by Streptomyces clavuligerus. J Ind Microbiol. 1988a; 3: 357-364.

Sánchez S, Chávez A, Forero A, García-Huante Y, Romero A, Sánchez M, et al. Carbon source regulation of antibiotic production. $J$ Antibiot (Tokyo). 2010; 63; 442-459.

Santamarta I, Pérez-Redondo R, Lorenzana LM, Martín JF, Liras P. Different proteins bind to butyrolactone receptor protein ARE sequence located upstream of the regulatory ccaR gene of Streptomyces clavuligerus. Mol Microbiol. 2005; 56: 824-835.

Sarada I, Sridhar P Nutritional improvement for cephamycin C fermentation using a superior strain of Streptomyces clavuligerus. Process Biochem. 1998; 33: 317-322

Zhu CH, Lu FP, He YN, Han ZL, Du LX. Regulation of avilamycin biosynthesis in Streptomyces viridochromogenes: effects of glucose, ammonium ion, and inorganic phosphate. Appl Microbiol Biotechnol. 2007; 73-10.
Received: October 10, 2011; Revised: December 19, 2011; Accepted: July 02, 2012. 


$$
\begin{aligned}
& \text { PAGINA } \\
& \text { EM } \\
& \text { BRANCO }
\end{aligned}
$$

